\title{
Полиморфные превращения и термическое расширение кристаллов $\mathrm{AgCuSe} \mathbf{0 . 5}_{0.5}(\mathrm{~S}, \mathrm{Te})_{0.5}$
}

\author{
(С) Ю.И. Алыев, Ю.Г. Асадов, Р.Д. Алыева, С.Г. Джабаров \\ Институт физики им. Г.М. Абдуллаева Национальной академии наук Азербайджана, \\ AZ-1073 Баку, Азербайджан \\ E-mail: yusifafshar@gmail.com, yusifasadov@rambler.ru, sakin@jinr.ru
}

(Получена 10 марта 2016 г. Принята к печати 7 ноября 2016 г.)

\begin{abstract}
Методом высокотемпературной рентгеновской дифрактометрии проведено исследование кристаллов состава $\mathrm{AgCuSe}_{0.5}(\mathrm{~S}, \mathrm{Te})_{0.5}$. Показано, что состав $\mathrm{AgCuSe}_{0.5} \mathrm{~S}_{0.5}$ при комнатной температуре состоит из $\mathrm{Cu}_{1.96} \mathrm{~S}$ и $\mathrm{AgCuSe}$. Эти фазы при $695 \mathrm{~K}$ превращаются в единую гранецентрированную кубическую фазу, превращение обратимо. Состав $\mathrm{AgCuSe}_{0.5} \mathrm{Te}_{0.5}$ при комнатной температуре трехфазный, включает $\mathrm{Cu}_{2} \mathrm{Te}, \mathrm{AgCuSe}$ и кубическую фазу. При $444 \mathrm{~K}$ обе орторомбические фазы одновременно превращаются в алмазоподобную кубическую фазу, при превращении кубическая фаза играет роль затравки. Из температурной зависимости параметров решетки рассчитаны коэффициенты теплового расширения существующих фаз в обоих составах по основным кристаллографическим направлениям.
\end{abstract}

DOI: $10.21883 /$ FTP.2017.06.44554.8233

\section{1. Введение}

Соединение $\mathrm{AgCuSe}$ образуется при соотношении компонентов $\mathrm{Ag}_{2} \mathrm{Se}: \mathrm{Cu}_{2} \mathrm{Se}=1: 1$ по перитектической реакции при температуре $1033 \mathrm{~K}$ [1]. Структура низкотемпературной фазы $\mathrm{AgCuSe}$ в $[2,3]$ определена как тетрагональная с параметрами решетки $a=4.083 \AA$, $c=6.30 \AA, Z=2, \quad$ пространственная группа (пр.гр.) $P 4 / n m m$, плотность $\rho=7.91 г / \mathrm{cm}^{3}$. Позднее в [4,5] кристаллическая структура $\mathrm{AgCuSe}$ определена как орторомбическая с параметрами решетки $a=4.105 \AA$, $b=20.350 \AA, c=6.310 \AA, Z=10, \rho=7.885$ г $/ \mathrm{cm}^{3}$. Последняя является сверхструктурой тетрагональной решетки. Параметр $b$ орторомбической решетки кратен 5 параметрам $b$ тетрагональной решетки. По данным [4], в орторомбической структуре $\mathrm{AgCuSe}$ атомы $\mathrm{Ag}$ находятся в плоскостях, перпендикулярных оси с. Около каждого из них располагаются 4 атома $\mathrm{Ag}$ на расстоянии $2.96 \AA$ и 6 атомов Se на расстояниях $2.67 \AA$ (4Se), $3.59 \AA$ (1Se), $3.64 \AA$ (1Se). Атомы Sе образуют вытянутые тетраэдры, в центре которых находятся атомы $\mathrm{Cu}$. Расстояния $\mathrm{Se}-\mathrm{Se}$ равны $3.03 \AA$, расстояния $\mathrm{Cu}-\mathrm{Se}$ равны 2.06-2.50 $\AA$, наименьшее расстояние $\mathrm{Cu}-\mathrm{Ag}$ составляет $2.98 \AA$.

В [6,7] показано, что орторомбическая модификация при $504 \mathrm{~K}$ превращается в высокотемпературную гранецентрированную кубическую (ГЦК) модификацию с параметром элементарной ячейки $a=6.0694 \AA$. Превращения в $\mathrm{AgCuSe}$ - обратимые.

В данной работе рассмотрены полиморфные превращения в $\mathrm{AgCuSe}_{0.5} \mathrm{~S}_{0.5}$ и $\mathrm{AgCuSe}_{0.5} \mathrm{Te}_{0.5}$.

\section{2. Экспериментальная часть}

Для формирования указанных составов выбрали метод прямого синтеза. В качестве реактора были применены двухстенные ампулы с внутренним диаметром 1 см и длиной 10 см из высококачественного кварца, который обладает большой термической стойкостью, в результате была обеспечена высокая герметичность, что исключает загрязнение и окисление синтезируемых веществ в случае растрескивания одной из двух ампул. Ампулы заполнялись исходными веществами $\mathrm{Ag}, \mathrm{Cu}, \mathrm{Se}, \mathrm{S}$ или Те в количествах, необходимых для каждого состава, эвакуировались до давления $10^{-3}$ Па, а затем запаивались. Участвующие исходные компоненты имели следующую чистоту: $\mathrm{Cu}$ - электролитическая, $\mathrm{Ag}$ - 99.999, S, Se и Те - марки В5. Ампулы с компонентами составов $\mathrm{AgCuSe}_{0.5} \mathrm{~S}_{0.5}$ и $\mathrm{AgCuSe}_{0.5} \mathrm{Te}_{0.5}$ помещали в печь и для предотвращения взрыва, а также для полной диффузии расплавленных селена, серы или теллура с серебром и медью температуру печи медленно повышали сначала до температуры плавления серы $\left(T_{m}=391 \mathrm{~K}\right)$, потом селена $\left(T_{m}=493 \mathrm{~K}\right)$, потом теллура $\left(T_{m}=725 \mathrm{~K}\right)$; ампулы выдерживали при этой температуре 4ч. После этого температуру печи повышали со скоростью $50 \mathrm{~K} /$ ч выше температуры плавления $\mathrm{AgCuSe}\left(T_{m}=1039 \mathrm{~K}\right)$. После выдержки в течение 3 ч при этой температуре с циклическим вибрированием ампулы с образцами медленно охлаждали до $400 \mathrm{~K}$, и при этой температуре с целью гомогенизации полученные образцы отжигались в течение 200 ч. Для установления структуры синтезированных образцов был проведен микроструктурный и рентгенофазовый анализ.

Порошкограмма от состава $\mathrm{AgCuSe}{ }_{0.5} \mathrm{~S}_{0.5}$, снятая при комнатной температуре, индицируется на основе параметров моноклинной решетки $\mathrm{Cu}_{1.96} \mathrm{~S}$ и орторомбической решетки $\mathrm{AgCuSe}$, т. е. при комнатной температуре состав $\mathrm{AgCuSe}{ }_{0.5} \mathrm{~S}_{0.5}$ оказывается двухфазным (табл. 1).

Анализ порошкограммы, полученной при комнатной температуре от состава $\mathrm{AgCuSe}_{0.5} \mathrm{Te}_{0.5}$, показал, что вещество состоит из трех фаз. Как видно из табл. 2, фаза I по размерам элементарной ячейки соответствует орторомбической фазе $\mathrm{Cu}_{2} \mathrm{Te}$, фаза II индицируется на 
Таблица 1. Расчет дифрактограмм $\mathrm{AgCuSe}_{0.5} \mathrm{~S}_{0.5}$ при различных температурах

\begin{tabular}{|c|c|c|c|c|c|c|c|c|}
\hline \multirow{2}{*}{$\begin{array}{c}T, \mathrm{~K} \\
\text { (эксп). }\end{array}$} & \multirow[b]{2}{*}{$\theta$} & \multirow[b]{2}{*}{$I / I_{0}$} & \multirow[b]{2}{*}{$\begin{array}{c}d, \AA \\
\text { (эксп.) }\end{array}$} & \multicolumn{2}{|c|}{$\mathrm{Cu}_{1.96} \mathrm{Se}$} & \multicolumn{2}{|c|}{$\mathrm{AgCuSe}$} & \multirow{2}{*}{$\begin{array}{c}\text { Параметры } \\
\text { кристаллической } \\
\text { решетки }\end{array}$} \\
\hline & & & & $\begin{array}{c}d, \AA \\
\text { (расч.) }\end{array}$ & $h k l$ & $\begin{array}{c}d, \AA \\
\text { (расч.) }\end{array}$ & $h k l$ & \\
\hline 1 & 2 & 3 & 4 & 5 & 6 & 7 & 8 & 9 \\
\hline 293 & $\begin{array}{l}18^{\circ} 00^{\prime} \\
19^{\circ} 30^{\prime} \\
20^{\circ} 24^{\prime} \\
21^{\circ} 36^{\prime} \\
23^{\circ} 30^{\prime} \\
25^{\circ} 06^{\prime} \\
25^{\circ} 36^{\prime} \\
26^{\circ} 36^{\prime} \\
28^{\circ} 24^{\prime} \\
31^{\circ} 24^{\prime} \\
33^{\circ} 42^{\prime} \\
37^{\circ} 24^{\prime} \\
39^{\circ} 06^{\prime} \\
41^{\circ} 24^{\prime} \\
45^{\circ} 54^{\prime} \\
48^{\circ} 00^{\prime}\end{array}$ & $\begin{array}{c}30 \\
80 \\
100 \\
50 \\
70 \\
40 \\
50 \\
60 \\
90 \\
40 \\
50 \\
10 \\
30 \\
10 \\
10 \\
20\end{array}$ & $\begin{array}{l}2.897 \\
2.682 \\
2.568 \\
2.432 \\
2.245 \\
2.111 \\
2.072 \\
1.999 \\
1.882 \\
1.718 \\
1.613 \\
1.474 \\
1.419 \\
1.354 \\
1.247 \\
1.205\end{array}$ & $\begin{array}{l}2.898 \\
2.690 \\
2.569 \\
2.437 \\
2.245 \\
2.111 \\
2.073 \\
1.998 \\
1.882 \\
1.717 \\
1.614 \\
1.473 \\
1.419 \\
1.354 \\
1.246 \\
1.205\end{array}$ & $\begin{array}{c}243 \\
10.0 .0 \\
044 \\
162 \\
942 \\
326 \\
11.2 .3 \\
645 \\
027 \\
275,390 \\
038 \\
908,286 \\
938 \\
078,1.0 .10 \\
0.5 .10 \\
0.6 .10\end{array}$ & $\begin{array}{c}2.890 \\
2.681 \\
- \\
- \\
- \\
- \\
- \\
2.012 \\
1.890 \\
1.710 \\
- \\
- \\
1.411 \\
- \\
1.247 \\
1.204\end{array}$ & $\begin{array}{c}150 \\
0.42 \\
- \\
- \\
- \\
- \\
- \\
220 \\
191 \\
0.10 .2 \\
- \\
- \\
243 \\
- \\
0.10 .4 \\
115\end{array}$ & $\begin{array}{c}\mathrm{Cu}_{1.96} \mathrm{~S} \\
\text { Моноклинная } \\
a=26.897 \AA \\
b=15.745 \AA \\
c=13.565 \AA \\
\beta=90.13^{\circ}, Z=128 \\
\text { пр. гр. } P 2_{1} / n \\
\rho=5.870 \text { г } / \mathrm{cm}^{3} \\
\text { АgСuSe } \\
\text { Орторомбическая } \\
a=4.086 \AA \\
b=20.533 \AA \\
c=6.275 \AA \\
Z=10 \\
\text { пр. гр. } P 4 / n m m \\
\rho=7.883 \text { г } / \mathrm{cm}^{3}\end{array}$ \\
\hline 373 & $\begin{array}{l}17^{\circ} 55^{\prime} \\
19^{\circ} 28^{\prime} \\
20^{\circ} 19^{\prime} \\
21^{\circ} 27^{\prime} \\
23^{\circ} 28^{\prime} \\
25^{\circ} 00^{\prime} \\
25^{\circ} 33^{\prime} \\
26^{\circ} 32^{\prime} \\
28^{\circ} 18^{\prime} \\
31^{\circ} 17^{\prime} \\
33^{\circ} 34^{\prime} \\
37^{\circ} 17^{\prime} \\
38^{\circ} 59^{\prime} \\
41^{\circ} 12^{\prime} \\
45^{\circ} 44^{\prime} \\
47^{\circ} 46^{\prime}\end{array}$ & $\begin{array}{r}30 \\
80 \\
100 \\
45 \\
70 \\
40 \\
50 \\
60 \\
90 \\
35 \\
45 \\
10 \\
25 \\
10 \\
10 \\
20\end{array}$ & $\begin{array}{l}2.909 \\
2.686 \\
2.578 \\
2.448 \\
2.248 \\
2.118 \\
2.075 \\
2.004 \\
1.888 \\
1.724 \\
1.619 \\
1.478 \\
1.423 \\
1.359 \\
1.250 \\
1.209\end{array}$ & $\begin{array}{l}2.910 \\
2.686 \\
2.579 \\
2.447 \\
2.248 \\
2.118 \\
2.074 \\
2.004 \\
1.888 \\
1.724 \\
1.619 \\
1.478 \\
1.423 \\
1.359 \\
1.250 \\
1.209\end{array}$ & $\begin{array}{c}243 \\
10.0 .0 \\
044 \\
162 \\
942 \\
326 \\
11.2 .3 \\
645 \\
027 \\
275,390 \\
038 \\
908 \\
938 \\
1.0 .10 \\
0.5 .10 \\
0.6 .10\end{array}$ & $\begin{array}{l}2.909 \\
2.684 \\
- \\
- \\
- \\
- \\
- \\
2.014 \\
1.906 \\
1.724 \\
- \\
- \\
1.411 \\
- \\
1.250 \\
1.201\end{array}$ & $\begin{array}{c}150 \\
042 \\
- \\
- \\
- \\
- \\
- \\
220 \\
191 \\
0.10 .2 \\
- \\
- \\
243 \\
- \\
0.10 .4 \\
115\end{array}$ & $\begin{array}{c}\mathrm{Cu}_{1.96} \mathrm{~S} \\
\text { Моноклинная } \\
a=26.856 \AA \\
b=15.811 \AA \\
c=13.608 \AA \\
\beta=90.13^{\circ}, Z=128 \\
\text { пр. гр. } P 2_{1} / n \\
\rho=5.759 \text { г } / \mathrm{cm}^{3} \\
\text { СuАgSe } \\
\text { Орторомбическая } \\
a=4.107 \AA \\
b=20.664 \AA \\
c=6.374 \AA \\
Z=10 \\
\text { пр. гр. } P 4 / n m m \\
\rho=7.672 \text { г } / \mathrm{cm}^{3}\end{array}$ \\
\hline 473 & $\begin{array}{l}17^{\circ} 49^{\prime} \\
19^{\circ} 26^{\prime} \\
20^{\circ} 10^{\prime} \\
21^{\circ} 22^{\prime} \\
23^{\circ} 24^{\prime} \\
24^{\circ} 45^{\prime} \\
25^{\circ} 27^{\prime} \\
26^{\circ} 30^{\prime} \\
28^{\circ} 00^{\prime} \\
31^{\circ} 05^{\prime} \\
33^{\circ} 12^{\prime} \\
37^{\circ} 17^{\prime} \\
38^{\circ} 59^{\prime} \\
41^{\circ} 12^{\prime} \\
45^{\circ} 44^{\prime} \\
47^{\circ} 46^{\prime}\end{array}$ & $\begin{array}{r}30 \\
75 \\
100 \\
50 \\
70 \\
40 \\
50 \\
60 \\
90 \\
40 \\
50 \\
10 \\
30 \\
10 \\
10 \\
20\end{array}$ & $\begin{array}{l}2.925 \\
2.691 \\
2.596 \\
2.456 \\
2.254 \\
2.138 \\
2.083 \\
2.006 \\
1.907 \\
1.734 \\
1.635 \\
1.490 \\
1.434 \\
1.374 \\
1.262 \\
1.220\end{array}$ & $\begin{array}{l}2.926 \\
2.691 \\
2.597 \\
2.456 \\
2.255 \\
2.138 \\
2.082 \\
2.006 \\
1.908 \\
1.734 \\
1.635 \\
1.490 \\
1.434 \\
1.374 \\
1.262 \\
1.221\end{array}$ & $\begin{array}{c}243 \\
10.0 .0 \\
044 \\
162 \\
942 \\
326 \\
11.2 .3 \\
645 \\
027 \\
275,390 \\
038 \\
908,286 \\
938 \\
078,1.0 .10 \\
0.50 .10 \\
0.6 .10\end{array}$ & $\begin{array}{l}2.925 \\
2.712 \\
- \\
- \\
- \\
- \\
- \\
2.030 \\
1.915 \\
1.734 \\
- \\
- \\
1.425 \\
- \\
1.262 \\
1.216\end{array}$ & $\begin{array}{c}150 \\
042 \\
- \\
- \\
- \\
- \\
- \\
220 \\
191 \\
0.10 .2 \\
- \\
- \\
243 \\
- \\
0.10 .4 \\
115\end{array}$ & 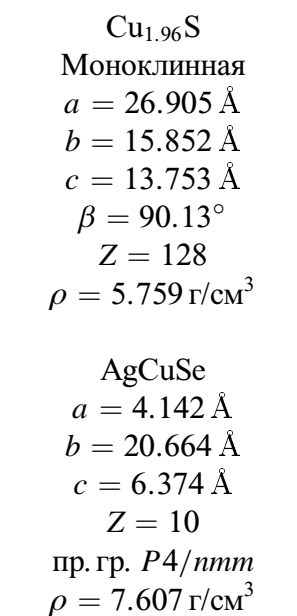 \\
\hline
\end{tabular}


Таблица 1 (продолжение).

\begin{tabular}{|c|c|c|c|c|c|c|c|c|}
\hline 1 & 2 & 3 & 4 & 5 & 6 & 7 & 8 & 9 \\
\hline 573 & $\begin{array}{l}17^{\circ} 47^{\prime} \\
19^{\circ} 24^{\prime} \\
20^{\circ} 07^{\prime} \\
21^{\circ} 20^{\prime} \\
23^{\circ} 22^{\prime} \\
24^{\circ} 42^{\prime} \\
25^{\circ} 25^{\prime} \\
26^{\circ} 17^{\prime} \\
27^{\circ} 56^{\prime} \\
31^{\circ} 02^{\prime} \\
33^{\circ} 08^{\prime} \\
36^{\circ} 51^{\prime} \\
38^{\circ} 32^{\prime} \\
40^{\circ} 35^{\prime} \\
45^{\circ} 06^{\prime} \\
47^{\circ} 06^{\prime}\end{array}$ & $\begin{array}{r}30 \\
80 \\
100 \\
45 \\
70 \\
40 \\
50 \\
60 \\
90 \\
35 \\
45 \\
10 \\
25 \\
10 \\
10 \\
20\end{array}$ & $\begin{array}{l}2.931 \\
2.694 \\
2.602 \\
2.460 \\
2.257 \\
2.142 \\
2.086 \\
2.022 \\
1.911 \\
1.736 \\
1.638 \\
1.493 \\
1.437 \\
1.376 \\
1.264 \\
1.222\end{array}$ & $\begin{array}{l}2.931 \\
2.694 \\
2.602 \\
2.460 \\
2.258 \\
2.142 \\
2.850 \\
2.021 \\
1.910 \\
1.737 \\
1.638 \\
1.493 \\
1.437 \\
1.376 \\
1.264 \\
1.222\end{array}$ & $\begin{array}{c}243 \\
10.0 .0 \\
044 \\
162 \\
942 \\
326 \\
11.2 .3 \\
645 \\
027 \\
275 \\
038 \\
908 \\
938 \\
1.0 .10 \\
0.0 .10 \\
0.6 .10\end{array}$ & $\begin{array}{c}2.931 \\
2.716 \\
- \\
- \\
- \\
- \\
- \\
2.039 \\
1.923 \\
1.741 \\
- \\
- \\
1.430 \\
- \\
1.266 \\
1.218\end{array}$ & $\begin{array}{c}150 \\
042 \\
- \\
- \\
- \\
- \\
- \\
220 \\
191 \\
0.10 .2 \\
- \\
- \\
243 \\
- \\
0.10 .4 \\
115\end{array}$ & $\begin{array}{c}\mathrm{Cu}_{1.96} \mathrm{~S} \\
a=26.936 \AA \\
b=15.893 \AA \\
c=13.775 \AA \\
\beta=90.13^{\circ} \\
Z=128 \\
\text { пр. гр. } P 2_{1} / n \\
\rho=5.643 \text { г/ } \mathrm{cm}^{3} \\
\\
\mathrm{AgCuSe} \\
a=4.152 \AA \\
b=20.695 \AA \\
c=6.383 \AA \\
Z=10 \\
\text { пр. гр. } P 4 / \mathrm{nmm}^{3} \\
\rho=5.567 \text { г } / \mathrm{cm}^{3}\end{array}$ \\
\hline 673 & $\begin{array}{l}17^{\circ} 44^{\prime} \\
19^{\circ} 23^{\prime} \\
20^{\circ} 05^{\prime} \\
21^{\circ} 15^{\prime} \\
23^{\circ} 20^{\prime} \\
24^{\circ} 40^{\prime} \\
25^{\circ} 24^{\prime} \\
26^{\circ} 15^{\prime} \\
27^{\circ} 54^{\prime} \\
30^{\circ} 57^{\prime} \\
33^{\circ} 05^{\prime} \\
36^{\circ} 48^{\prime} \\
38^{\circ} 29^{\prime} \\
40^{\circ} 32^{\prime} \\
45^{\circ} 01^{\prime} \\
47^{\circ} 00^{\prime}\end{array}$ & $\begin{array}{r}30 \\
80 \\
100 \\
50 \\
70 \\
40 \\
50 \\
60 \\
90 \\
40 \\
50 \\
10 \\
30 \\
10 \\
10 \\
20\end{array}$ & $\begin{array}{l}2.937 \\
2.696 \\
2.606 \\
2.466 \\
2.260 \\
2.145 \\
2.087 \\
2.024 \\
1.913 \\
1.740 \\
1.640 \\
1.494 \\
1.438 \\
1.377 \\
1.266 \\
1.224\end{array}$ & $\begin{array}{l}2.937 \\
2.696 \\
2.606 \\
2.467 \\
2.260 \\
2.145 \\
2.087 \\
2.024 \\
1.913 \\
1.740 \\
1.640 \\
1.494 \\
1.438 \\
1.377 \\
1.266 \\
1.224\end{array}$ & $\begin{array}{c}243 \\
10.0 .0 \\
044 \\
162 \\
942 \\
326 \\
11.2 .3 \\
645 \\
027 \\
275 \\
038 \\
908 \\
938 \\
1.0 .10 \\
0.5 .10 \\
0.6 .10\end{array}$ & $\begin{array}{c}2.939 \\
2.720 \\
- \\
- \\
- \\
- \\
- \\
2.039 \\
1.923 \\
1.741 \\
- \\
- \\
1.430 \\
- \\
1.266 \\
1.220\end{array}$ & $\begin{array}{c}150 \\
042 \\
- \\
- \\
- \\
- \\
- \\
220 \\
191 \\
0.10 .2 \\
- \\
- \\
243 \\
- \\
0.10 .4 \\
115\end{array}$ & $\begin{array}{c}\mathrm{Cu}_{1.96} \mathrm{~S} \\
a=26.963 \AA \\
b=15.921 \AA \\
c=13.793 \AA \\
\beta=90.13^{\circ} \\
Z=128 \\
\text { пр. гр. } P 2_{1} / n \\
\rho=5.620 \text { г } / \mathrm{cm}^{3} \\
\\
\text { AgCuSe } \\
a=4.160 \AA \\
b=20.757 \AA \\
c=6.387 \AA \\
Z=10 \\
\text { пр. гр. } P 4 / \mathrm{nmm} \\
\rho=7.525 \Gamma / \mathrm{cm}^{3}\end{array}$ \\
\hline 773 & $\begin{array}{l}16^{\circ} 19^{\prime} \\
23^{\circ} 30^{\prime} \\
27^{\circ} 52^{\prime} \\
29^{\circ} 12^{\prime} \\
34^{\circ} 19^{\prime} \\
37^{\circ} 54^{\prime} \\
39^{\circ} 04^{\prime} \\
43^{\circ} 39^{\prime} \\
47^{\circ} 04^{\prime}\end{array}$ & $\begin{array}{r}40 \\
65 \\
100 \\
70 \\
30 \\
80 \\
30 \\
50 \\
90\end{array}$ & $\begin{array}{l}3.176 \\
2.246 \\
1.915 \\
1.834 \\
1.588 \\
1.457 \\
1.420 \\
1.297 \\
1.222\end{array}$ & $\begin{array}{l}3.185 \\
2.245 \\
1.915 \\
1.835 \\
1.588 \\
1.458 \\
1.420 \\
1.297 \\
1.223\end{array}$ & $\begin{array}{c}200 \\
220 \\
311 \\
222 \\
400 \\
331 \\
420 \\
422 \\
333,511\end{array}$ & & & $\begin{array}{c}\text { ГЦК } \\
\mathrm{AgCuSe}_{0.5} \mathrm{~S}_{0.5} \\
a=6.356 \AA \\
\text { пр. гр. } F m 3 m \\
Z=4 \\
\rho=6.376 \text { г } / \mathrm{cm}^{3}\end{array}$ \\
\hline 873 & $\begin{array}{l}16^{\circ} 16^{\prime} \\
23^{\circ} 27^{\prime} \\
27^{\circ} 49^{\prime} \\
29^{\circ} 10^{\prime} \\
34^{\circ} 16^{\prime} \\
37^{\circ} 51^{\prime} \\
39^{\circ} 02^{\prime} \\
43^{\circ} 36^{\prime} \\
47^{\circ} 01^{\prime}\end{array}$ & $\begin{array}{r}40 \\
65 \\
100 \\
70 \\
30 \\
80 \\
30 \\
50 \\
90 \\
\end{array}$ & $\begin{array}{l}3.196 \\
2.250 \\
1.918 \\
1.837 \\
1.590 \\
1.459 \\
1.422 \\
1.298 \\
1.225\end{array}$ & $\begin{array}{l}3.182 \\
2.250 \\
1.919 \\
1.837 \\
1.591 \\
1.460 \\
1.423 \\
1.299 \\
1.225\end{array}$ & $\begin{array}{l}200 \\
220 \\
311 \\
222 \\
400 \\
311 \\
420 \\
422 \\
333\end{array}$ & & & $\begin{array}{c}\text { ГЦК } \\
\mathrm{AgCuSe}_{0.5} \mathrm{~S}_{0.5} \\
a=6.363 \AA \\
\text { пр. гр. Fm3m } \\
Z=4 \\
\rho=6.355 \text { г } / \mathrm{cm}^{3}\end{array}$ \\
\hline 973 & $\begin{array}{l}16^{\circ} 13^{\prime} \\
23^{\circ} 24^{\prime} \\
27^{\circ} 46^{\prime} \\
29^{\circ} 07^{\prime} \\
34^{\circ} 13^{\prime} \\
37^{\circ} 49^{\prime} \\
39^{\circ} 00^{\prime} \\
43^{\circ} 33^{\prime} \\
46^{\circ} 58^{\prime}\end{array}$ & $\begin{array}{r}40 \\
65 \\
100 \\
70 \\
30 \\
80 \\
30 \\
50 \\
90\end{array}$ & $\begin{array}{l}3.205 \\
2.254 \\
1.921 \\
1.840 \\
1.592 \\
1.460 \\
1.422 \\
1.299 \\
1.225\end{array}$ & $\begin{array}{l}3.186 \\
2.253 \\
1.921 \\
1.840 \\
1.593 \\
1.462 \\
1.425 \\
1.301 \\
1.226\end{array}$ & $\begin{array}{l}200 \\
220 \\
311 \\
222 \\
400 \\
331 \\
420 \\
422 \\
333\end{array}$ & & & $\begin{array}{c}\text { ГЦК } \\
\mathrm{AgCuSe}_{0.5} \mathrm{~S}_{0.5} \\
a=6.372 \AA \\
\text { пр. гр. Fm } 3 m \\
Z=4 \\
\rho=6.328 \text { г } / \mathrm{cm}^{3}\end{array}$ \\
\hline
\end{tabular}

Примечание. Режим измерения: излучение $\mathrm{Cu} K_{\alpha}$ (длина волны $1.5418 \AA$ ), фильтр $-\mathrm{Ni}, 35$ кВ, 10 мА. I/I0 - относительные интенсивности, эксп. - эксперимент, расч. - расчет. 
Таблица 2. Расчет дифрактограммы $\mathrm{AgCuSe}_{0.5} \mathrm{Te}_{0.5}$ при различных температурах

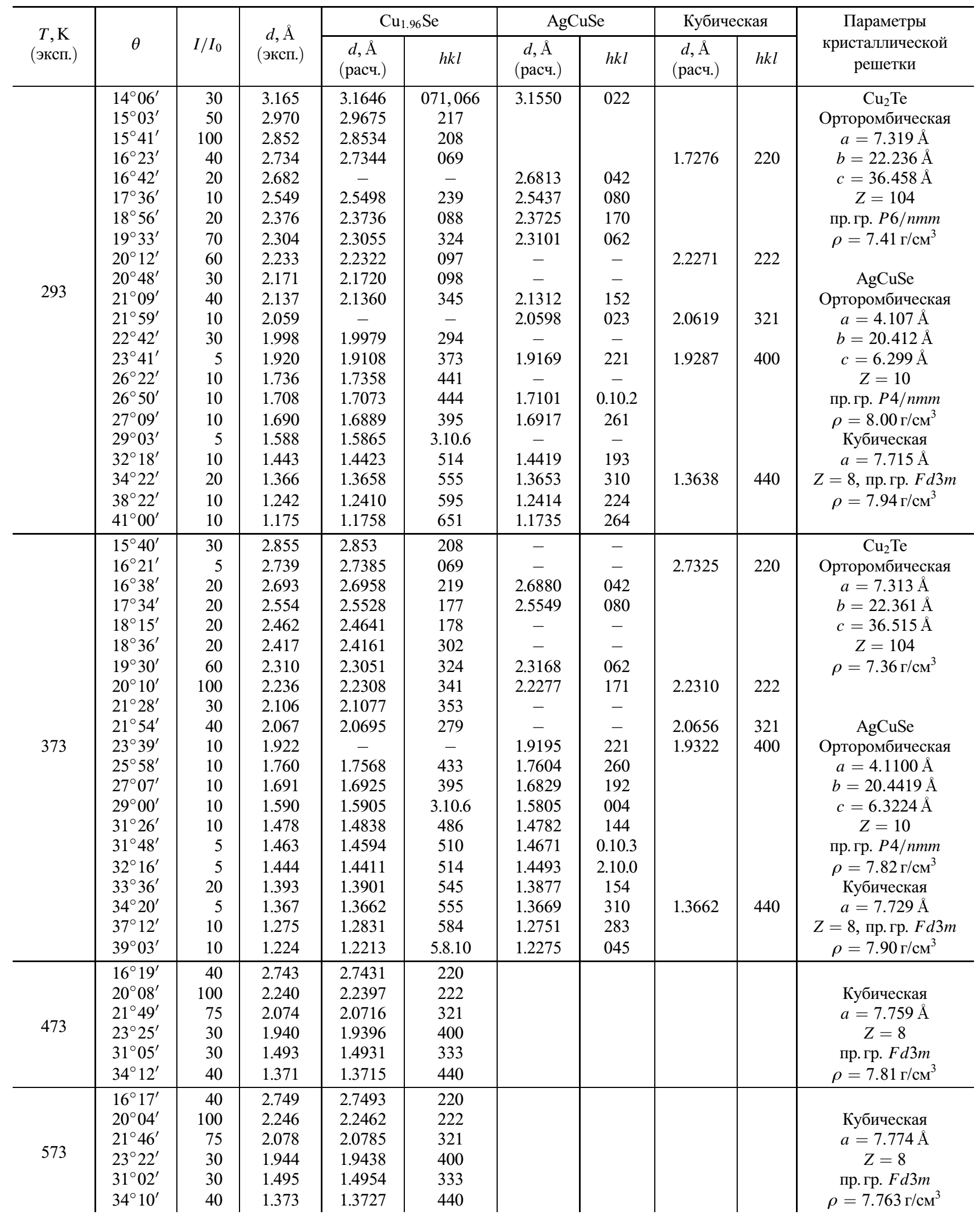

Примечание. Режим измерения: излучение $\mathrm{Cu} K_{\alpha}$ (длина волны $1.5418 \AA$ ), фильтр $-\mathrm{Ni}, 35$ кВ, 10 мА. I/I 0 - относительные интенсивности, эксп. - эксперимент, расч. - расчет. 
основе параметров орторомбической решетки $\mathrm{AgCuSe}$ и фаза III при комнатной температуре соответствует метастабильно существующей кубической фазе в структуре типа алмаза.

Для получения монокристаллов $\mathrm{AgCuSe}_{0.5} \mathrm{~S}_{0.5}$ и $\mathrm{AgCuSe}_{0.5} \mathrm{Te}_{0.5}$ был применен метод Бриджмена [8] c добавлением третьей температурной зоны. Синтезированные поликристаллические вещества погружались в измельченном виде в ампулы (специально изготовленные для метода Бриджмена) длиной 10 см и внутренним диаметром $1 \mathrm{~cm}$. Эвакуированные до давления $10^{-3}$ Па ампулы помещались в верхней зоне печи, температура которой регулировалась с помощью терморегулятора. В первой зоне температуру печи подняли выше температуры плавления $\mathrm{AgCuSe}$. После выдержки в течение 3 ч при указанной температуре включался электромотор, при помощи которого ампула опускалась со скоростью $0.2 \mathrm{cм} /$ ч.

При переходе вещества в ампуле через температуру плавления начинают расти кристаллы высокотемпературной модификации, с понижением температуры образуются кристаллы низкотемпературной модификации и растут внутри монокристалла высокотемпературной модификации. Монокристалличность этой модификации зависит в основном от разности плотностей матричного $\left(\rho_{1}\right)$ и растущего $\left(\rho_{2}\right)$ кристалла: при $\rho_{1} \approx \rho_{2}$ происходят превращения типа монокристалл-монокристалл; при $\rho_{1} \gg \rho_{2}$ и $\rho_{1} \leq \rho_{2}$ происходят превращения типа монокристалл-поликристалл. Из такого поликристалла методом полиморфных превращений получение монокристаллов невозможно.

Поэтому монокристалличность низкотемпературной модификации не зависит от выбранного метода. При понижении температуры за счет процесса упорядочения от единого монокристалла высокотемпературной модификации выделяется несколько фаз, различающихся составом и структурой.

Исследования при высоких температурах проводились на дифрактометре ДРОН-3М $\left(\mathrm{Cu} K_{\alpha}\right.$-излучение, длина волны $1.5418 \AA$, Ni-фильтр) с высокотемпературной приставкой URVT-2000 в вакууме, при остаточном давлении $10^{-2}$ Па. Условие разрешения записи составляло $\sim 0.1^{\circ}$. Использовали режим непрерывного сканирования. В экспериментах ошибка определения дифракционных углов $\theta$ не превышала $\Delta \theta= \pm 0.02^{\circ}$.

\section{3. Обсуждение результатов}

$\mathrm{AgCuSe}_{0.5} \mathrm{~S}_{\mathbf{0 . 5}}$. Для изучения полиморфных превращений в $\mathrm{AgCuSe} \mathrm{f}_{0.5} \mathrm{~S}_{0.5}$ при комнатной температуре изготовили образец размером $4 \times 4 \times 2$ мм. В интервале углов $10 \leq 2 \theta \leq 100^{\circ}$ были зарегистрированы 16 дифракционных отражений (табл. 1), анализ которых подтверждает существование в образце двух фаз, одна из которых по размерам элементарной ячейки идентична низкотемпературной моноклинной фазе $\mathrm{Cu}_{1.96} \mathrm{~S}$ с параметра-

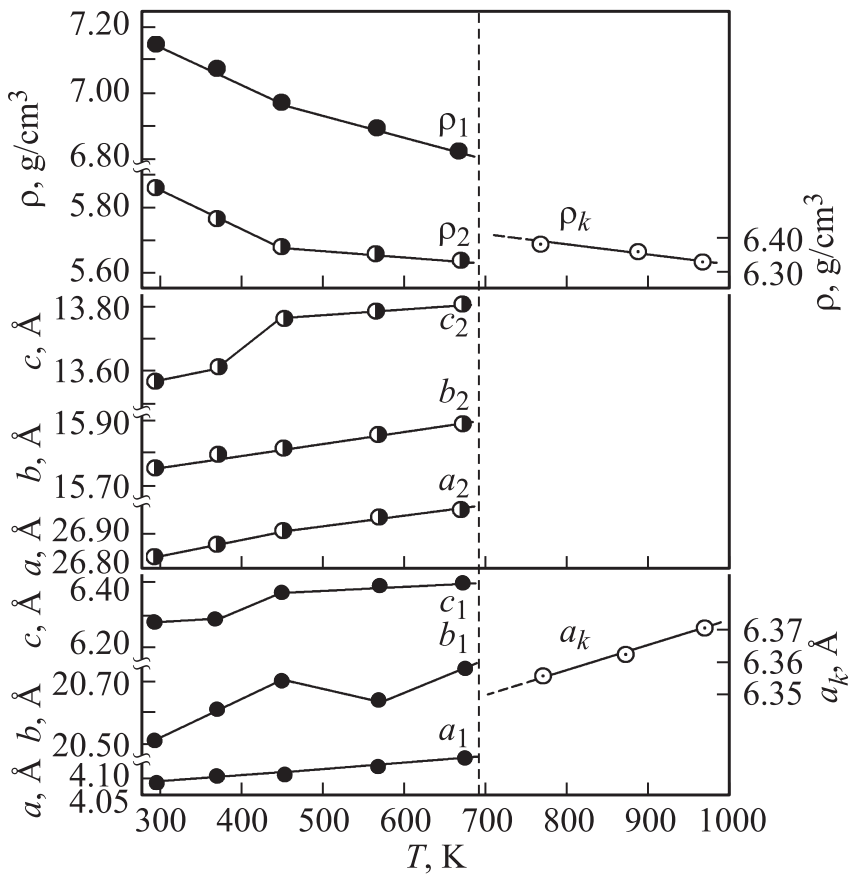

Рис. 1. Температурные зависимости параметров кристаллической решетки $(a, b, c)$ и плотности $(\rho)$ существующих фаз в $\mathrm{AgCuSe}_{0.5} \mathrm{~S}_{0.5}$ : орторомбическая фаза $\mathrm{AgCuSe}-a_{1}, b_{1}, c_{1}, \rho_{1}$; моноклинная фаза $\mathrm{Cu}_{1.6} \mathrm{~S}-a_{2}, b_{2}, c_{2}, \rho_{2}$; кубическая фаза $a_{k}, \rho_{k}$.

ми решетки $a=26.897 \AA, b=15.745 \AA, c=13.565 \AA$, $\beta=90.13^{\circ}, Z=128$, пр.гр. $P 21 / n[6]$, а другая имеет элементарную ячейку низкотемпературной орторомбической фазы $\mathrm{AgCuSe}$ с параметрами $a=4.086 \AA$, $b=20.533 \AA, c=6.275 \AA, Z=10$, пр. гр. $P 4 / n m m$ [4].

После записи дифракции при комнатной температуре, не нарушая ориентации кристалла, включали печь и через каждые $100 \mathrm{~K}$ проводили контрольные записи. Надо отметить, что с ростом температуры число отражений и их интенсивности, зарегистрированные при комнатной температуре, остаются постоянными. Только при $773 \mathrm{~K}$ в прежнем интервале углов регистрируются 9 новых отражений, принадлежащих высокотемпературной ГЦК модификации с параметром элементарной ячейки $a=6.356 \AA, Z=4$, пр. гр. $F m 3 m, \rho=6.376$ г $/ \mathrm{cm}^{3}$ (табл. 1). Установлено, что температура равновесия между низкотемпературной и высокотемпературной модификациями составляет $T_{0}=695 \mathrm{~K}$.

В интервале температур 293-973 K рассчитаны параметры решетки существующих модификаций в $\mathrm{AgCuSe}_{0.5} \mathrm{~S}_{0.5}$, которые приведены в табл. 1 и графически на рис. 1. Как видно из рис. 1 , параметр $a_{1}$ орторомбической и параметры $a_{2}$ и $b_{2}$ моноклинной фаз в интервале температур 293-673 K растут линейно, а параметр $b_{1}$ уменьшается в интервале от 473 до $573 \mathrm{~K}$ и после этой температуры растет. Параметр $c_{1}$ орторомбической и параметр $c_{2}$ моноклинной фаз при $473 \mathrm{~K}$ отклоняются от линейности и после $473 \mathrm{~K}$ растут линейно. Несмотря 
Таблица 3. Тепловое расширение кристалла $\mathrm{AgCuSe} \mathrm{F}_{0.5} \mathrm{~S}_{0.5}$

\begin{tabular}{c|c|c|c|c|c|c}
\hline Состав & $T, \mathrm{~K}$ & $\alpha_{[100]}$ & $\alpha_{[010]}$ & $\alpha_{[001]}$ & $\bar{\alpha}=\frac{\alpha_{[100]}+\alpha_{[010]}+\alpha_{[001]}}{3}$ & $\beta=\alpha_{[100]}+\alpha_{[010]}+\alpha_{[001]}$ \\
\hline & $293-373$ & 52.007 & 55.423 & 17.917 & 41.782 & 125.347 \\
$\mathrm{AgCuSe}$ & $293-473$ & 66.623 & 47.370 & 83.170 & 65.721 & 197.163 \\
& $293-573$ & 45.451 & 27.320 & 61.998 & 44.923 & 134.769 \\
& $293-673$ & 49.592 & 28.080 & 47.778 & 41.817 & 125.450 \\
\hline & $293-373$ & 18.638 & 38.901 & 42.388 & 33.309 & 99.927 \\
$\mathrm{Cu}_{1.96} \mathrm{~S}$ & $293-473$ & 17.810 & 23.288 & 81.091 & 40.730 & 122.189 \\
& $293-573$ & 16.109 & 23.137 & 57.132 & 32.126 & 96.378 \\
& $293-673$ & 14.126 & 23.232 & 46.365 & 27.908 & 83.723 \\
\hline \multirow{2}{*}{$\mathrm{AgCuSe}_{0.5} \mathrm{~S}_{0.5}$} & $773-873$ & 11.013 & & & 11.013 & 33.039 \\
& $773-973$ & 12.587 & & & 12.587 & 37.761
\end{tabular}

Примечание. $\alpha_{[100]}, \alpha_{[010]}, \alpha_{[001]}-$ коэффициенты теплового расширения по направлениям [100], [010], [001] в единицах 10-6 $\mathrm{K}^{-1}$.

Таблица 4. Коэффициенты теплового расширения (в $\left.10^{-6} \mathrm{~K}^{-1}\right)$ кристалла $\mathrm{AgCuSe}_{0.5} \mathrm{Te}_{0.5}$

\begin{tabular}{c|c|c|c|c|c|c}
\hline Состав & $T, \mathrm{~K}$ & $\alpha_{[100]}$ & $\alpha_{[010]}$ & $\alpha_{[001]}$ & $\bar{\alpha}=\frac{\alpha_{[100]}+\alpha_{[010]}+\alpha_{[001]}}{3}$ & $\beta=\alpha_{[100]}+\alpha_{[010]}+\alpha_{[001]}$ \\
\hline $\mathrm{Cu}_{2} \mathrm{Te}$ & $293-373$ & 17.079 & 70.268 & 24.605 & 37.317 & 111.951 \\
\hline $\mathrm{AgCuSe}$ & $293-373$ & 9.310 & 12.854 & 45.642 & 22.542 & 67.627 \\
\hline $\mathrm{AgCuSe}_{0.5} \mathrm{~S}_{0.5}$, & $293-373$ & 22.683 & & & 22.683 & 68.049 \\
кубическая & $293-473$ & 31.684 & & & 31.684 & 95.052 \\
& $293-573$ & 27.312 & & & 27.312 & 81.936
\end{tabular}

на такое поведение параметров решетки обеих модификаций в зависимости от температуры, в числах дифракционных отражений и их интенсивностях существенных изменений не происходит.

Рассчитанные из температурной зависимости параметров решетки существующих модификаций в составе $\mathrm{AgCuSe}{ }_{0.5} \mathrm{~S}_{0.5}$ коэффициенты теплового расширения $\alpha$ приведены в табл. 3. Как видно из таблицы, в модификации, соответствующей структуре $\mathrm{Cu}_{1.96} \mathrm{~S}$, тепловое расширение в направлении [010] заметно отличается от [100] и [001], т. е. $\alpha_{[100]}<\alpha_{[010]}>\alpha_{[001]}$.

Кристаллы $\mathrm{AgCuSe}{ }_{0.5} \mathrm{~S}_{0.5}$, состоящие из двух фаз при комнатной температуре, при повышении температуры, как видно из анизотропии теплового расширения, деформируют друг друга. На границе раздела этих фаз образуется зародыш ГЦК модификации, который растет за счет этих фаз. Из единой ГЦК модификации при охлаждении выделяются две фазы, т.е. кристалл возвращается в первоначальное состояние.

$\mathrm{AgCuSe}_{0.5} \mathrm{Te}_{\mathbf{0 . 5}}$. При комнатной температуре от произвольно ориентированного состава $\mathrm{AgCuSe}_{0.5} \mathrm{Te}_{0.5}$ в интервале углов $10 \leq 2 \theta \leq 90^{\circ}$ были зарегистрированы 22 дифракционных отражения, которые совпали с отражениями, зарегистрированными от порошкограммы синтезированного образца (табл. 2). Как видно из табл. 2, $\mathrm{AgCuSe}_{0.5} \mathrm{Te}_{0.5}$ при комнатной температуре состоит из трех фаз: фаза I по размерам элементарной ячейки идентична низкотемпературной орторомбической $\mathrm{Cu}_{2} \mathrm{Te}$ с параметрами кристаллической решетки $a=7.319 \AA$, $b=22.236 \AA, c=36.458 \AA$, фаза II обладает элементарной ячейкой, отвечающей низкотемпературной орторомбической модификации $\mathrm{AgCuSe}$ и кубическая фаза III имеет алмазаподобную структуру с параметром решетки $a=7.319 \AA$.

На дифрактограмме большинство рефлексов от наблюдаемых трех фаз накладываются друг на друга. Причиной многофазности состава $\mathrm{AgCuSe}_{0.5} \mathrm{Te}_{0.5}$ в основном является перераспределение анионов ( $\mathrm{Se}, \mathrm{Te})$ и катионов $(\mathrm{Ag}, \mathrm{Cu})$. После записи дифрактограммы при комнатной температуре включали печь и через каждые $50 \mathrm{~K}$ проводили контрольные записи. Температуру образца перед началом каждой записи поддерживали постоянной в течение 40 мин. При этих условиях остается трехфазность образца $\mathrm{AgCuSe}_{0.5} \mathrm{Te}_{0.5}$, и при $444 \mathrm{~K}$ обе орторомбические фазы превращаются в кубическую фазу (см. табл. 2). Превращение обратимое, т.е. при охлаждении образец возвращается в первоначальное состояние.

На рис. 2 приведены температурные зависимости параметров решетки существующих фаз в $\mathrm{AgCuSe}_{0.5} \mathrm{Te}_{0.5}$ в интервале температур $293-573 \mathrm{~K}$. Из рис. 2 видно, что параметры решетки обеих орторомбических и кубической фаз в зависимости от температуры растут линейно. При превращении обеих орторомбических фаз в кубическую температурная зависимость параметра $a=f(T)$ не отклоняется от линейности. Отсюда следует, что ку- 


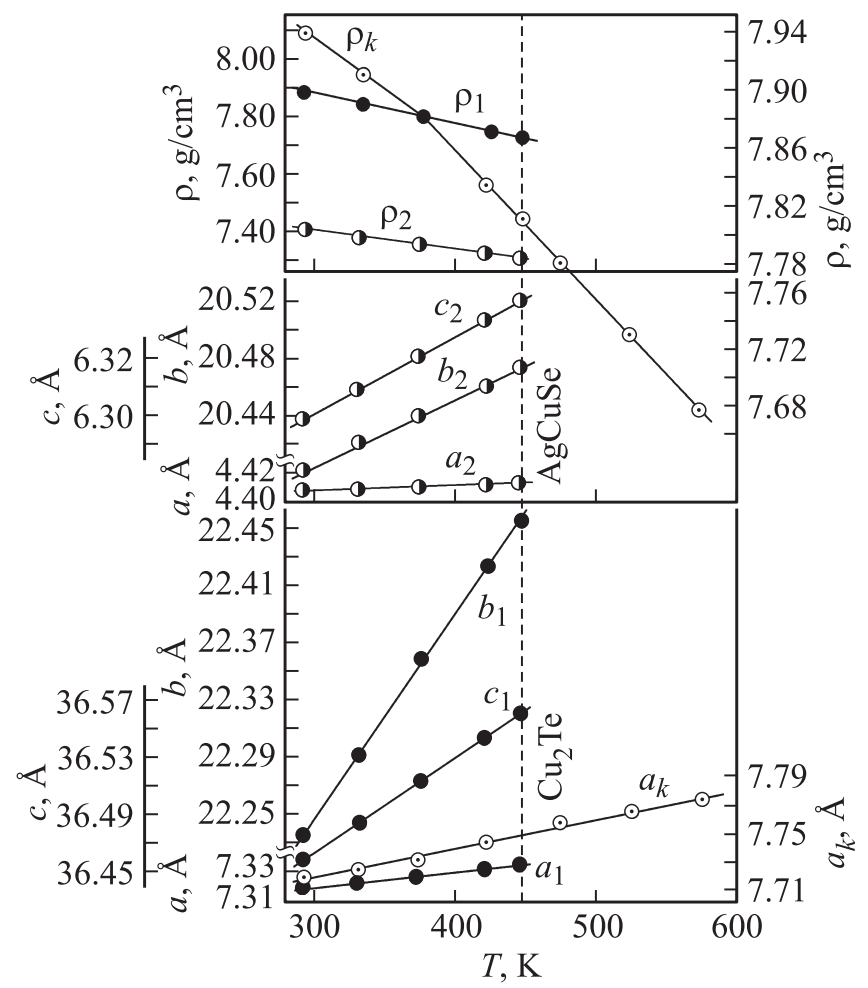

Рис. 2. Температурные зависимости параметров кристаллической решетки $(a, b, c)$ и плотности $(\rho)$ существующих фаз в $\mathrm{AgCuSe}_{0.5} \mathrm{Te}_{0.5}$ : орторомбическая фаза $\mathrm{Cu}_{2} \mathrm{Te}-a_{1}, b_{1}, c_{1}, \rho_{1}$; моноклинная фаза $\mathrm{AgCuSe}-a_{2}, b_{2}, c_{2}, \rho_{2}$; кубическая модификация $-a_{k}, \rho_{k}$.

бическая фаза при превращении обеих орторомбических фаз играет роль готовой затравки.

Из температурной зависимости параметров решетки рассчитаны коэффициенты теплового расширения обеих орторомбических и кубических фаз, которые представлены в табл. 4.

Как видно из табл. 4, коэффициенты теплового расширения в обеих орторомбических фазах по основным кристаллографическим направлениям имеют сильную анизотропию. Это является одной из основных причин нестабильности структуры обеих орторомбических фаз.

\section{4. Заключение}

Кристаллы $\mathrm{AgCuSe}$ при комнатной температуре имеют орторомбические структуры. Замещение половины анионов Se атомами $\mathrm{S}$ приводит к появлению $\mathrm{Cu}_{1.96} \mathrm{~S}$ и $\mathrm{AgCuSe}$, а при замещении половины анионов $\mathrm{Se}$ теллуром кристалл становится трехфазным, т.е. имеются $\mathrm{AgCuSe}, \mathrm{Cu}_{2} \mathrm{Te}$ и кубическая фазы. При температуpe $695 \mathrm{~K} \mathrm{Cu}_{1.96} \mathrm{~S}$ и $\mathrm{AgCuSe}$ превращаются в единую ГЦК фазу, а $\mathrm{Cu}_{2} \mathrm{Te}$ и $\mathrm{AgCuSe}$ при $444 \mathrm{~K}$ превращаются в алмазоподобную кубическую фазу. В обоих случаях превращения обратимые.

\section{Список литературы}

[1] М.Н. Агаев, Ш.М. Алекперова, М.И. Заргарова. Докл. АН АзССР, 27 (5), 20 (1971).

[2] N.N. Bikkulova, G.N. Asylguzhina, A.N. Skomorokhov, Yu.M. Stepanov. Bull. Rus. Acad. Sci. Phys., 70 (4), 641 (2006).

[3] I.W. Earley. Amer. Mineralogist., 35 (5-6), 345 (1950).

[4] A.J. Frueh, G.K. Czamanke, C.H. Knight. Z. Krist., 108, 389 (1957).

[5] Y.G. Asadov, Y.I. Aliyev, A.G. Babaev. Phys. Part. Nucl., 46 (3), 452 (2015).

[6] Ш.К. Кязымов, Г.Ш. Гасанов, Ю.Г. Асадов. Докл. АН АзСCP, 41 (11), 33 (1986).

[7] R.B. Baikulov, Yu.G. Asadov. Inorg. Mater., 41 (4), 338 (2005).

[8] A.L.N. Stevels, F. Jellinek. Rec. Trav. Chim., 90 (3), 273 (1971).

Редактор Л.В. Шаронова

\section{Polymorphous transformations and thermal expansion of $\mathrm{AgCuSe}_{0.5}(\mathrm{~S}, \mathrm{Te})_{0.5}$}

Yu.I. Alyev, Yu.G. Asadov, R.D. Alyeva, S.G. Dzhabarov Institute of Physics,

National Academy of Sciences of Azerbaijan, AZ-1073 Baku, Azerbaijan 